Copyright 2013 American Institute of Physics. This article may be downloaded for personal use only. Any other use requires prior permission of the author and the American Institute of Physics.

The following article appeared in Appl. Phys. Lett. 192213112 (2013) and may be found at http://dx.doi.org/10.1063/1.4807663 


\section{AIP Applied physises Letters}

\section{lon-beam-induced bending of freestanding amorphous nanowires: The importance of the substrate material and charging}

Ajuan Cui, J. C. Fenton, Wuxia Li, Tiehan H. Shen, Zhe Liu et al.

Citation: Appl. Phys. Lett. 102, 213112 (2013); doi: 10.1063/1.4807663

View online: http://dx.doi.org/10.1063/1.4807663

View Table of Contents: http://apl.aip.org/resource/1/APPLAB/v102/i21

Published by the AIP Publishing LLC.

Additional information on Appl. Phys. Lett.

Journal Homepage: http://apl.aip.org/

Journal Information: http://apl.aip.org/about/about_the_journal

Top downloads: http://apl.aip.org/features/most_downloaded

Information for Authors: http://apl.aip.org/authors

\section{ADVERTISEMENT}

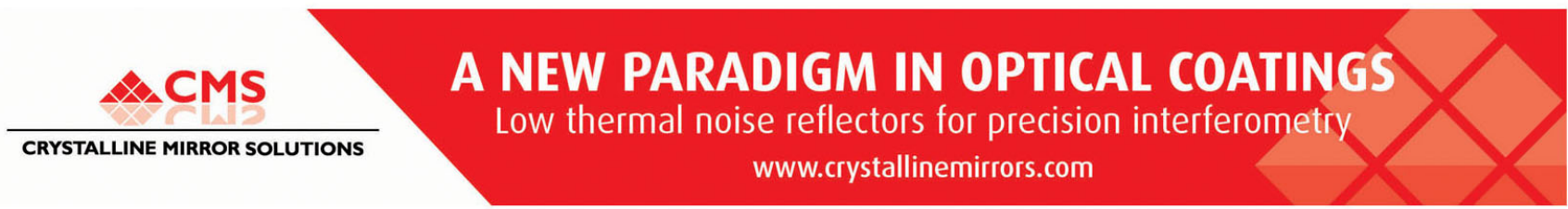




\title{
lon-beam-induced bending of freestanding amorphous nanowires: The importance of the substrate material and charging
}

\author{
Ajuan Cui, ${ }^{1, a)}$ J. C. Fenton, ${ }^{2}$ Wuxia Li ${ }^{1, b)}$ Tiehan H. Shen, ${ }^{3}$ Zhe Liu, ${ }^{1}$ Qiang Luo, ${ }^{1}$ \\ and Changzhi Gu ${ }^{1, b)}$ \\ ${ }^{1}$ Beijing National Laboratory of Condensed Matter Physics, Institute of Physics, Chinese Academy of Sciences, \\ Beijing 100190, China \\ ${ }^{2}$ London Centre for Nanotechnology, University College London, London WC1E 7JE, United Kingdom \\ ${ }^{3}$ Joule Physics Laboratory, University of Salford, Manchester M5 4WT, United Kingdom
}

(Received 21 March 2013; accepted 7 May 2013; published online 29 May 2013)

\begin{abstract}
Ion-beam irradiation offers great flexibility and controllability in the construction of freestanding nanostructures with multiple advanced functionalities. Here, we present and discuss the bending of free-standing nanowires, against, towards, and ultimately parallel to a flux of directional ion irradiation. Bending components both along and perpendicular to the incident ion beam were observed, and the bending behavior was found to depend both on the ion beam scanning strategy and on the conductivity of the supporting substrate. This behavior is explained by an ion-irradiation-related electrostatic interaction. Our findings suggest the prospect of exploiting this technique to engineer 3D nanostructures for advanced applications. (C) 2013 AIP Publishing LLC. [http://dx.doi.org/10.1063/1.4807663]
\end{abstract}

In recent years, much attention has been paid to threedimensional nanostructures as building blocks for a wide range of future nanoscale devices, exhibiting novel electronic, ${ }^{1}$ opti$\mathrm{cal}^{2}{ }^{2}$ magnetic, ${ }^{3}$ and mechanical properties. ${ }^{4}$ However, preparation of $3 \mathrm{D}$ nanoscale structures following a top-down approach is not very practical. ${ }^{5}$ For a bottom-up approach involving the construction of freestanding nanodevices, bending of nanowires by ion-beam irradiation has recently been intensively investigated. This technique can be used to precisely tailor the shape of a freestanding building block. ${ }^{6-18}$ To date, the study of ion-beam-irradiation-assisted bending has been conducted on nanowires of a wide range of materials, including carbon nanotube/nanowires, ${ }^{6-8} \mathrm{Si}_{3} \mathrm{~N}_{4}, \mathrm{Au} / \mathrm{Si}_{3} \mathrm{~N}_{4}$, $\mathrm{MoSi}_{2}$ cantilevers, ${ }^{9-11} \mathrm{Si}^{12}{ }^{12} \mathrm{GaAs},{ }^{13} \mathrm{ZnO},{ }^{14}$ and Ge. ${ }^{15} \mathrm{~A}$ number of bending behaviours have been observed and different bending mechanisms have been proposed, ${ }^{72-17}$ taking into consideration parameters such as the kinetic energy, mass, fluence, and incident angle of the ion beam. However, little attention has been given to the possible effect of Coulomb interaction and the role the substrate plays in this. ${ }^{6-17}$

In the present work, we explore the effect of the supporting-substrate conductivity and ion-beam scanning strategy on the bending induced by focused-ion-beam irradiation in freestanding amorphous nanowires. We observe the bending of the nanowires towards, away from, and transverse to the ion beam depending on the substrate used and the details of the ion scans. We present a hypothesis that the bending behavior of the wires is significantly influenced by charges deposited on the substrate as the ion beam is scanned in the vicinity of the nanowires. A simple calculation is described which suggests that these electrostatic forces are of the correct order of magnitude to account for the mediation of the observed bending. The results demonstrate that the strategy employed for scanning the

\footnotetext{
a) E-mail: cuiajuan@aphy.iphy.ac.cn

b) Authors to whom correspondence should be addressed. Electronic addresses: liwuxia@aphy.iphy.ac.cn and czgu@aphy.iphy.ac.cn
}

ion beam may be used to control the orientation of selected free-standing nanowires and has potential application in postgrowth shape manipulation of free-standing nanostructures for advanced nanodevice fabrication.

The $\mathrm{W}$ nanowires used in this study were grown by FIBinduced deposition using a $1 \mathrm{pA}$ ion beam current on various substrates with $\mathrm{W}(\mathrm{CO})_{6}$ as gas precursor. The growth details can be found in our previous work. ${ }^{17}$ Single-frame ion beam scans were performed over areas containing these nanowires and repeated successively, the incident angle (angle between the normal to the substrate and the ion beam) being kept fixed at $50^{\circ}$, the beam energy at $30 \mathrm{keV}$, and ion beam currents in the range of $40 \mathrm{pA}-150 \mathrm{pA}$. The scanning speed and hence the irradiation time were also varied in some of the experiments. A raster pattern was used, consisting of 884 lines in a single frame, with the beam scanning from left to right and then top to bottom. After each scan, the sample stage was tilted to $45^{\circ}$ off normal to the electron beam to enable in situ scanning electron microscopic (SEM) observation of the nanowires and to measure the amount of bending.

First, the effect of the underlying substrate on the bending of FIB-grown $\mathrm{W}$ nanowires was studied. Three sorts of substrates were used: $\mathrm{Al}$ substrates, and $\mathrm{SiO}_{2}$ $(200 \mathrm{~nm}) / \mathrm{Si}$ substrates supporting Au pads with thickness $\sim 65 \mathrm{~nm}$ (each pad with an area of $30 \mu \mathrm{m} \times 30 \mu \mathrm{m}$ ). Freestanding nanowires with $70 \mathrm{~nm}$ radius and $3.0 \mu \mathrm{m}$ height were grown. Fig. 1(a) shows SEM images after successive ion sweeps of $\mathrm{W}$ wires grown on an $\mathrm{Al}$ substrate. The nanowires initially bent towards the ion beam and, after sufficient ion-beam irradiation, aligned with the ion beam; see Fig. 1(b). By contrast, when $\mathrm{Au} / \mathrm{SiO}_{2} / \mathrm{Si}$ substrates were used, the $\mathrm{W}$ nanowires with radius of $105 \mathrm{~nm}$ first bent away from the ion beam before bending back towards the ion beam and finally aligning with the ion beam (Figs. 2(a) and 2(b)). To find out whether the switching of the bending direction was caused by the difference in size or the electric conductivity of nanowires when different substrates were used, the same 


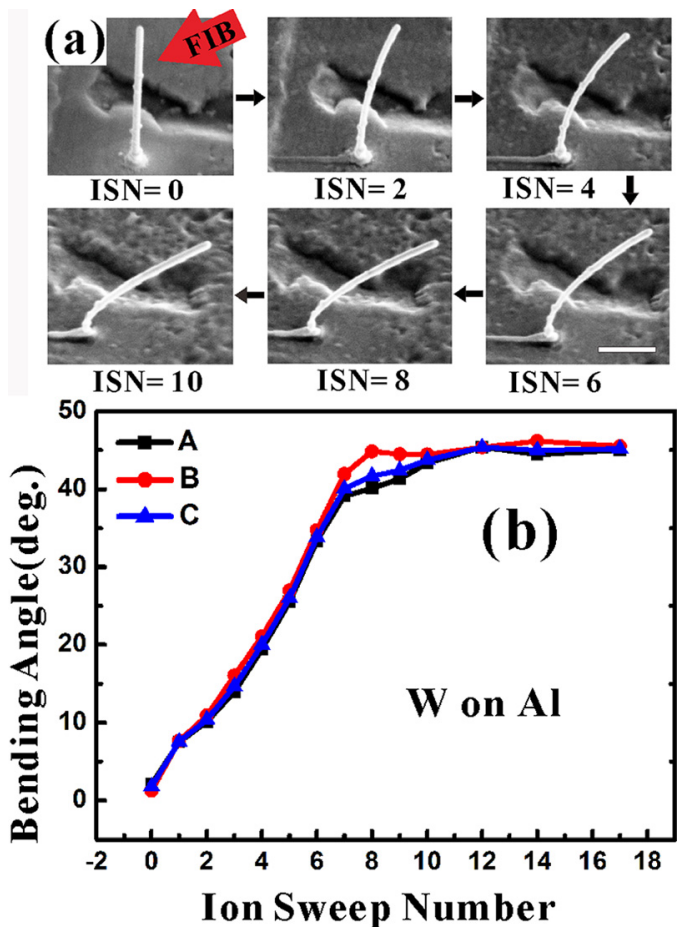

FIG. 1. Bending of FIB-grown W nanowires grown on Al substrates: (a) SEM images and (b) the bending angle as a function of the ion beam sweep number. The scale bar is $1 \mu \mathrm{m}$; the lines are guides to the eye.

experiment was conducted on FIB-grown platinum nanowires, which were $106 \mathrm{~nm}$ in radius and have a resistivity of about 5 times larger than the tungsten nanowires. Again, Pt nanowires grown on $\mathrm{Al}$ substrate only bent towards the ion beam though those grown on $\mathrm{Au} / \mathrm{SiO}_{2} / \mathrm{Si}$ substrates were first bent away from the ion beam, then bent back towards the ion beam. These observations confirm that the geometry of the irradiated nanowires is not a contributing factor that leads to the changing of the bending direction. Moreover, unlike nanowires grown on $\mathrm{Al}$ substrates, which ultimately aligned with the incident ion beam and maintained that position even when further ion sweeps were performed, nanowires grown on $\mathrm{Au} / \mathrm{SiO}_{2} / \mathrm{Si}$ substrates often broke at the base before they were completely aligned with the incident ion beam. This is likely to be associated with milling of the base of the nanowire weakening it at this point. Note that Fig. 2(a) also shows that the Au pad on the substrate is gradually removed during the milling.

The bending trajectory of a nanowire can also be influenced by changing the position of the ion-beam scanning area with respect to the nanowires being irradiated. Using reduced-raster scanning or by moving the sample stage, a nanowire can be placed in the center or at one side of the ion-beam scanning area. The sample position was extremely carefully adjusted to lie at the coincident point, the point at which the SEM and FIB beams cross. Fig. 3(a) illustrates the relative position of the incident ion beam to the nanowires. $\mathrm{X}$ and $\mathrm{Y}$ label the perpendicular directions in the substrate plane; the ion-beam is directed towards the nanowire from an angle above the $+\mathrm{Y}$ axis and, for SEM imaging, the electron beam is incident at $45^{\circ}$ to the $-\mathrm{Y}$ axis.

For nanowires grown on $\mathrm{Al}$ substrates, when a sufficiently high ion-dose was used, bending occurred only along the $+\mathrm{Y}$ direction (towards the incident ion beam). However,
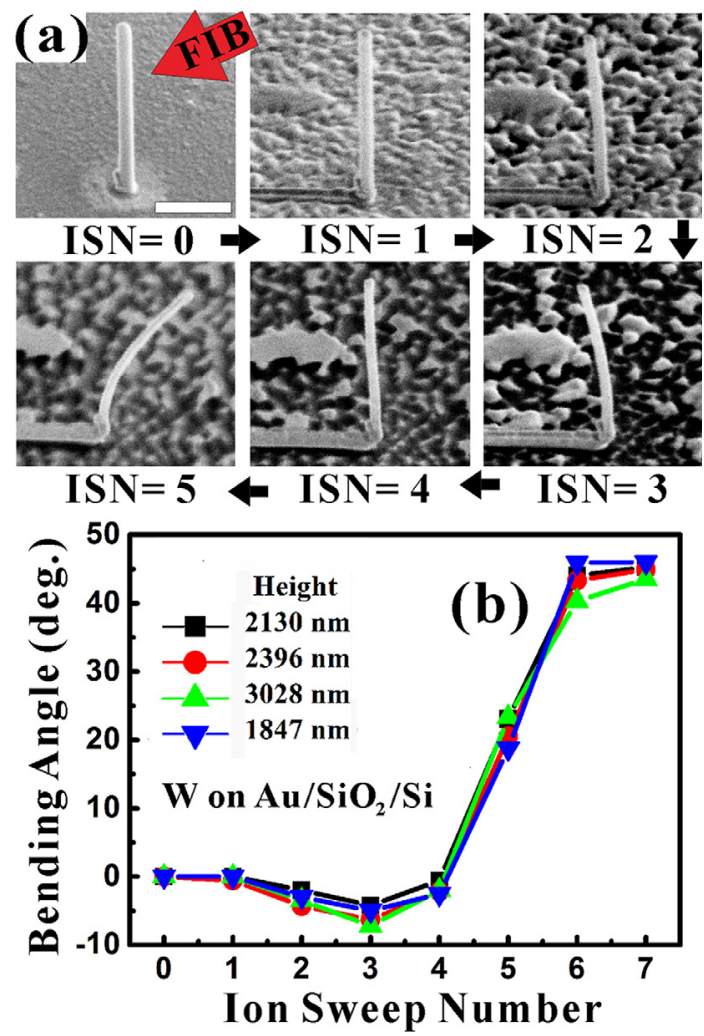

FIG. 2. Bending of FIB-grown W nanowires grown on isolated $30 \mu \mathrm{m}$ square $\mathrm{Au}$ pads $\left(30 \mathrm{~nm}\right.$ thick) on $\mathrm{SiO}_{2}(200 \mathrm{~nm}) / \mathrm{Si}$ substrates: (a) $\mathrm{SEM}$ images and (b) the bending angle as a function of the ion-beam sweep number. The ion beam current was $47 \mathrm{pA}$. The scan covered an area of $15.2 \times 13.1 \mu \mathrm{m}^{2}$ on the substrate and took $163 \mathrm{~s}$. The scale bar is $1 \mu \mathrm{m}$; the lines are guides to the eye.

when the nanowire was standing on an insulating substrate, a bending component in the $-\mathrm{X}$ or $+\mathrm{X}$ direction could also arise, dependent on the relative position of the nanowire within the ion beam scanning area (shown in Fig. 3(b)) and (revealingly) also on the scanning speed: when a $150 \mathrm{pA}$ ion beam was scanned rapidly across an area of $15.2 \times 13.1 \mu \mathrm{m}^{2}$, taking $6 \mathrm{~s}$ for a single scan, bending with a component in the $-\mathrm{X}$ or $+\mathrm{X}$ direction was observed, as can be seen from the
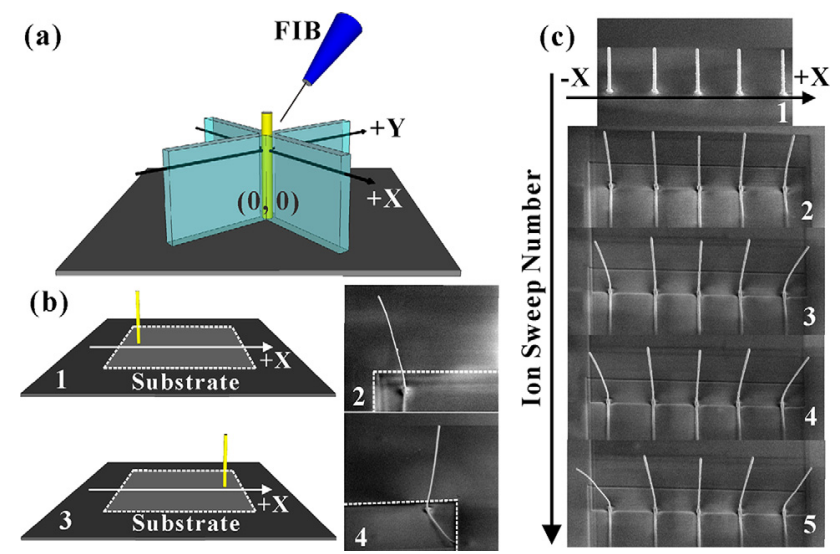

FIG. 3. Scanning-strategy-dependent bending phenomena: (a) schematic diagram illustrating the position of the nanowire relative to the incident ion beam; (b) the dependence of the bending direction on the relative location of wires in the ion-beam scanning area; (c) bending images of $\mathrm{W}$ nanowires grown on $\mathrm{SiO}_{2} / \mathrm{Si}$ substrates taken on successive sweeps indicate bending in both $+\mathrm{Y}$ $(-Y)$ and $+\mathrm{X}(-\mathrm{X})$ directions for wires near the edge of the ion-beam scanning area. The electron beam scans from $-\mathrm{X}$ to $+\mathrm{X}$ and from $+\mathrm{Y}$ to $-\mathrm{Y}$. Note that the ion-beam scans are from $+\mathrm{X}$ to $-\mathrm{X}$ and from $-\mathrm{Y}$ to $+\mathrm{Y}$. 
SEM images taken on successive ion sweeps over a group of 5 nanowires shown in Fig. 3(c). Bending in the direction normal to the incident ion beam is clearly seen: For the nanowire at the left edge of the irradiated area, the wire initially bent towards the $-\mathrm{X},-\mathrm{Y}$ quadrant (image 2 of Fig. 3(b)); when the wire was in the center of the ion beam scanning area, it first bent along $-\mathrm{Y}$ and then bent back along $+\mathrm{Y}$ and finally aligned with the incident ion beam. When the nanowire was on the right side of the ion beam scanning area, it first bent towards the $+\mathrm{X},-\mathrm{Y}$ quadrant (image 4 of Fig. 3(b)), then to the $+\mathrm{X},+\mathrm{Y}$ quadrant, and finally aligned with the ion beam.

By contrast, when a slower ion-beam scan speed, taking $163 \mathrm{~s}$ to scan the same area, was used, no obvious bending along the $+\mathrm{X}$ or $-\mathrm{X}$ direction was observed: these wires only bent along the $-\mathrm{Y}$ and $+\mathrm{Y}$ directions. This bending in one direction due to a slow scan could be reversed by a subsequent fast scan and then restored by another slow scan. Following a fast scan $(6 s)$ over the same area, the nanowire was observed to be bent in a particular direction, dependent on the location of the irradiated nanowire (as discussed above). After an additional irradiation using a slow scan $(163 \mathrm{~s})$ of the same scanning area, the nanowire returned to its original position. After this switching had been performed several times, no further bending along the $+\mathrm{X}$ or $-\mathrm{X}$ direction was found; this change coincided with our expectations about when the surface $\mathrm{SiO}_{2}$ layer on the substrate has been removed during the prolonged ion irradiation.

The bending behavior observed in $\mathrm{W}$ nanowires grown on $\mathrm{SiO}_{2} / \mathrm{Si}$ and $\mathrm{Au} / \mathrm{SiO}_{2} / \mathrm{Si}$ substrates, namely first bending away and then towards the ion beam, is similar to the observations reported on crystalline $\mathrm{Si}$ and $\mathrm{Ge}$ nanowires ${ }^{12,15}$ on $\mathrm{Si}$ substrates, which was explained as a gradual amorphization, starting from the side facing the ion beam until the process was completed across the wire. Our W composite nanowires as deposited were amorphous in nature and therefore our observations cannot be accounted for by the same explanation. Various features of the results we have observed suggest that electrostatic forces may mediate the bending process described here. The difference in bending behavior for wires of the same material scanned over the same-size area using the same current but grown on substrates with differing surface resistivity $\left(9.4 \times 10^{-5} \Omega / \square\right.$ and $5.0 \times 10^{23} \Omega / \square$ for $\mathrm{Al}$ and $\mathrm{SiO}_{2}$, respectively) is suggestive of the importance of charging and, in Fig. 3, the only clear differences between the situations of the wires on either sides of the scan area-showing opposite behavior-are most likely to be charge-related. The presence of charge deposited on the surface of the substrate during an ion scan produces a force on the nanowire in the direction in which bending is indeed observed in Fig. 2(a): The nanowire becomes positively charged as a result of irradiation by the positively charged ion beam and the substrate also becomes positively charged. While these charges persist, there is, therefore, a net force on the nanowire away from the center of the ion linescan. The symmetrical effect of scanning on wires near the left edge of the scan area and those near the right edge of the area implies that the behavior is not determined by a force between the charged ion-beam itself and the (perhaps charged) nanowire. Note that a $30 \mathrm{pA} 30 \mathrm{keV}$ ion beam contains at any instant $\sim 1$ ion per $\mathrm{mm}$ and this explains why electrostatic forces involving the ion beam itself are not important.
The persistence of the bends after the ion scan has finished shows that although bends due to electrostatic forces may be elastic initially, they become plastic at some stage, most likely during or shortly after the ion beam strikes the nanowire. ${ }^{20}$ It is likely that the energy deposited in the wire in collisions leads to structural changes in the vicinity of the collision site, which relax strain in the wire and tend to fix the bend in the nanowire, corresponding to an increased (decreased) likelihood of material ending up in regions of tensile (compressive) strain.

The expected elastic bend of a nanowire as a result of the electrostatic forces was calculated by using a simple model. Charge deposited on the substrate leaks away over some time characterized by the conductivity of the substrate surface. For a substrate with sufficiently low surface-conductivity, we can model the charge deposited on the substrate as a line charge. The net force on these charges in directions perpendicular to the nanowire leads to a transverse deflection $d$, which may be determined from the theory of bending beams ${ }^{21}$ as

$$
d=-\frac{W}{6 E l} b^{2}(3 l-b),
$$

where $W$ is the net transverse force and $b$ is the distance from the base of the nanowire (of length $l$, Young's modulus $E$, and area moment of inertia $I$ ).

Taking $E=150 \mathrm{GPa}^{22}$ the wires to be of circular crosssection, neglecting secondary electron emission ${ }^{19}$ and allowing charges to move along the nanowire in response to both the external electrostatic force and the mutual repulsion between deposited charges, we arrive at a net deflection which depends on the location of the line charge with respect to the nanowire. Fig. 4(a) shows how the deflection of the wire resulting from a single linescan depends on the location of the wire along the linescan and on the height above the base at which the ion-beam strikes the wire. The nanowire deflects away from the center of the linescan and by a greater amount further away from the center of the linescan, the same as observed in the experiments with insulating substrates and the same order of magnitude. The deflection
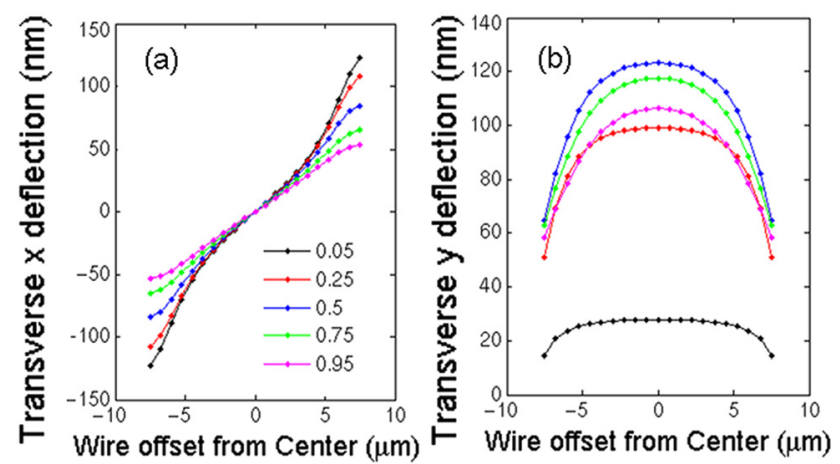

FIG. 4. Deflection expected in (a) the $\mathrm{X}$ direction and (b) the $\mathrm{Y}$ direction as a result of repulsion between the charge deposited on the nanowire and the line of charge deposited on the substrate by a single linescan, as a function of the offset of the nanowire from the center of the linescan. A wire of length $3 \mu \mathrm{m}$ and radius $100 \mathrm{~nm}$ is considered, with a $6 \mathrm{~s}$ frame scan consisting of linescans of $30 \mu \mathrm{m}$ and flyback time of $60 \mathrm{~ns}$ per line, with a $47 \mathrm{pA}$ beam current incident at an angle of $40^{\circ}$ above the substrate. The different curves depict the deflection resulting from scans at different fractions up the nanowire from its base. 
increases in proportion to the number of linescans for which charge persists on the substrate. The difference in bending behavior between fast and slow scans suggests that this timescale for charge to leak away is $\sim 10-100 \mathrm{~ms}^{23}$

This electrostatic force also has a component in the $+\mathrm{Y}$ direction. Fig. 4(b) shows the expected deflection resulting, which is the same order of magnitude as the transverse bending and is largest when the nanowire is close to the center of the linescan and for linescans around halfway up the nanowire; this is associated with the directionality and the fall-off trend of the electrostatic interaction.

For a conducting substrate, charge deposited on either the wire or on the substrate leaks away rapidly so we should expect the magnitudes of the electrostatic forces at least to be much smaller. For the isolated $\mathrm{Au}$ pad on $\mathrm{SiO}_{2} / \mathrm{Si}$ substrate, until the Au layer is removed, the charge accumulated on the Au pad will quickly become uniformly distributed over the pad and, therefore, a net electrostatic force bending the nanowires away from the center of the pad is expected. However, the observations do not seem to reflect the bending expected from such a force. The proposed model of electrostatic interaction cannot explain why the wires eventually have the tendency to bend towards the ion beam direction. This could perhaps be associated with compressive strain in the wires produced by ion modification of the exposed surface of the wires. ${ }^{13}$ Whilst the precise mechanism for such a plastic deformation that "homes in" on the direction of the ion beam is not clear, it is clear that there is some competing force that eventually wins over the Coulomb interaction. This broad interpretation agrees with the observed approximate coincidence of the change in the bending direction of the wires on the gold pads and the removal of the gold layer and, shortly after, the $\mathrm{SiO}_{2}$ insulating layer: subsequent to this, the wires are on a conductive substrate so that the accumulation of charge on the surface and associated Coulomb interaction with the wire is no longer significant. Whilst the electrostatic-interaction-mediated bending is clearly dependent on the detailed model of the charge distribution profile on the surface, the simple model presented certainly illustrates that qualitatively it appeared to agree with the overall trends observed experimentally.

We should emphasize that the electrostatic interaction alone cannot produce a plastic deformation, so the role of the ion beam in providing local heating/annealing and structural modification is pivotal and is less well understood. Momentum transfer of the ions to the nanowire leads to a force away from the ion beam. The magnitude of the force on the wire due to momentum transfer should cause to a deflection of the wire $\sim 1 \mathrm{~nm}$. If a substantial proportion of this bend is fixed into the wire on each linescan, the total bend might reach several tens of $\mathrm{nm}$, large enough to explain the bending away from the ion beam. The modification of the exposed area of the wire surface-layer by the ion beam may also be a significant factor; phenomena of crystalline amorphization, implanting of the incident ion species, and sputtering of the irradiated material may all be relevant. ${ }^{24}$ Nevertheless, a careful calibration of processing parameters is likely to make the electrostatic-mediated ion-beaminduced nanowire bending a practical tool for nanoscale manipulation.
In conclusion, we have systematically investigated postgrowth shape manipulation of freestanding amorphous nanowires using FIB irradiation. We have found that a highly reproducible and controllable bending behavior is dependent on the ion-beam scanning strategy and also on the conductivity of the supporting substrate, suggesting electrostatic interactions are involved in the mediation of the ion irradiation induced nanowire bending. This is of potential use as a technique for nanostructure fabrication and manipulation. A further study of the precise nature of the processes is to be conducted in the near future.

The authors would like to acknowledge helpful conversations with Huan Wang and Dr. Paul Warburton, Dr. Grenville Jones, and Professor Philip Grundy. This work was supported by the National Natural Science Foundation of China under Grant Nos. 91123004, 11104334, 50825206, 60871045, and 10834012, the Outstanding Technical Talent Program of the Chinese Academy of Sciences, and the National Basic Research Program (973) of China under Grant No. 2009CB930502.

${ }^{1}$ B. Tian, T. Cohen-Karni, Q. Qing, X. Duan, P. Xie, and C. M. Lieber, Science 329, 830 (2010).

${ }^{2}$ J. Valentine, S. Zhang, T. Zentgraf, E. Ulin-Avila, D. A. Genov, G. Bartal, and X. Zhang, Nature 455, 376 (2008).

${ }^{3}$ E. J. Romans, E. J. Osley, L. Young, P. A. Warburton, and W. Li, Appl. Phys. Lett. 97, 222506 (2010).

${ }^{4}$ S. Hu, L. Eberhard, J. Chen, J. C. Love, J. P. Butler, J. J. Fredberg, G. M. Whitesides, and N. Wang, Am J Physiol Cell Physiol. 287(5), C1184-C1191 (2004).

${ }^{5}$ J. Stang, V. Holy, and G. Bauer, Rev. Mod. Phys. 76, 725 (2004).

${ }^{6}$ T. Morita, R. Kometani, K. Watanabe, K. Kanda, Y. Haruyama, T. Hoshino, K. Kondo, T. Kaito, T. Ichihashi, J. Fujita, M. Ishida, Y. Ochiai, T. Tajima, and S. Matsui, J. Vac. Sci. Technol. B 21(6), 2737 (2003).

${ }^{7}$ S. K. Tripathi, N. Shukla, S. Dhamodaran, and V. N. Kulkarni, Nanotechnology 19, 205302 (2008).

${ }^{8}$ B. C. Park, K. Y. Jung, W. Y. Song, O. Beom-Hoan, and S. J. Ahn, Adv. Mater. 18, 95 (2006).

${ }^{9}$ W. J. Arora, S. Sijbrandij, L. Stern, J. Notte, H. I. Smith, and G. Barbastathis, J. Vac. Sci. Technol. B 25, 2184 (2007).

${ }^{10}$ L. Xia, W. Wu, J. Xu, Y. Hao, and Y. Wang, Proc. IEEE Micro. Elect. 22, 118 (2006).

${ }^{11}$ T. Yoshida, M. Nagao, and S. Kanemaru, Jpn. J. Appl. Phys., Part 1 49, 056501 (2010).

${ }^{12}$ K. Jun, J. Joo, and J. M. Jacobson, J. Vac. Sci. Technol. B 27, 3043 (2009).

${ }^{13}$ C. Borschel, R. Niepelt, S. Geburt, C. Gutsche, I. Regolin, W. Prost, F.-J. Tegude, D. Stichtenoth, D. Schwen, and C. Ronning, Small 5, 2576-2580 (2009).

${ }^{14}$ C. Borschel, S. Spindler, D. Lerose, A. Bochmann, S. H. Christiansen, S. Nietzsche, M. Oertel, and C. Ronning, Nanotechnology 22, 185307 (2011).

${ }^{15}$ L. Romano, N. G. Rudawski, M. R. Holzworth, K. S. Jones, S. G. Choi, and S. T. Picraux, J. Appl. Phys. 106, 114316 (2009).

${ }^{16}$ T. I. Kamins, R. S. Williams, T. Hesjedal, and J. S. Harris, Physica E 13, 995 (2002).

${ }^{17}$ A. J. Cui, W. X. Li, Q. Luo, Z. Liu, and C. Z. Gu, Microelectron. Eng. 98, 409 (2012).

${ }^{18}$ A. Cui, W. Li, Q. Luo, Z. Liu, and C. Z. Gu, Appl. Phys. Lett. 100, 143106 (2012).

${ }^{19}$ N. Yao, Focused Ion Beam Systems-Basic and Application (Cambridge University, Cambridge, England, 2007).

${ }^{20}$ K. Mitsuishi, M. Shimojo, M. Takeguchi, M. Tanaka, and K. Furuya, Jpn. J. Appl. Phys., Part 1 45, 5517 (2006).

${ }^{21}$ W. C. Young, R. G. Budynas, and A. M. Sadegh, Roark's Formulas for Stress and Strain, 8th ed. (The McGraw-Hill Companies, Inc., New York, NY, 2012).

${ }^{22}$ H. Wang, Ph.D. dissertation, University College London, 2013.

${ }^{23}$ N. A. Kislov and I. I. Khodos, Microsc. Microanal. Microstruct. 3, 323-331 (1992).

${ }^{24}$ I. Utkea, P. Hoffmann, and J. Melngailis, J. Vac. Sci. Technol. B. 26, 1197 (2008). 